\title{
El Test de Actitudes hacia la Alimentación en Costa Rica: primeras evidencias de validez y confiabilidad
}

\author{
The Eating Attitude Test in Costa Rica: \\ first evidences of its validity and reliability
}

\author{
Zaida Salazar Mora \\ Escuela de Psicología, Universidad de Costa Rica
}

\author{
Dirección postal: Escuela de Psicología, Universidad de Costa Rica \\ 11501-2060 San José \\ Ce: zaidasalazar@hotmail.com
}

\begin{abstract}
Resumen. Objetivo. Se analiza la estructura factorial y la confiabilidad del Test de Actitudes hacia la Alimentación (EAT-40) y se examina su efectividad para diferenciar a las personas diagnosticadas con trastornos de la conducta alimentaria (TCA: anorexia y bulimia). Participantes. Participaron en el estudio 569 mujeres entre los 10 y los 39 años de edad con y sin diagnóstico de TCA ( $n=19, n=533$, respectivamente), quienes llenaron el cuestionario EAT40. Procedimientos. En una primera parte se realizó el análisis factorial confirmatorio de la escala, luego se estimó la confiabilidad interna de cada subescala, seguidamente se examinó la validez de criterio mediante la comparación de los grupos con y sin diagnóstico de TCA. Resultados. Las estadísticas psicométricas mostraron resultados satisfactorios a nivel de la estructura factorial (tres factores) y alfa de Cronbach $(\alpha=.889)$; asimismo, se obtuvieron buenas propiedades de discriminación a partir de los puntos de corte de 30 (sensibilidad 63.2\%, especificidad $85.8 \%$ ) y de 20 (sensibilidad $73.7 \%$, especificidad $71.8 \%$ ) y se evidenció que el EAT-40 diferencia entre personas con y sin diagnóstico de TCA.
\end{abstract}

Palabras clave: Test de Actitudes hacia la Alimentación, trastornos de la conducta alimentaria, propiedades psicométricas, validez, confiabilidad.

\begin{abstract}
Purpose: The factorial structure and reliability of the Eating Attitudes Test (EAT-40) were analyzed. The ability of the test to discriminate subjects with eating disorder (ED: anorexia and bulimia) diagnosis was also examined. Participants. Five hundred sixty nine women between 10 and 39 years old with and without diagnostic of eating disorder $(n=19, n$ $=533$, respectively) completed the EAT-40. Procedures. In a first step, confirmatory factor analyses were conducted, followed by the calculation of the internal consistency of the subscales. Secondly, criterion validity was assessed by testing differences across groups with and without eating disorder diagnosis. Results. Psychometric analyses showed adequate factorial structure (three factors) and internal consistency $(\alpha=.889)$. Data also showed satisfactory discrimination properties of the measure at the cut points of 30 (sensibility $63.2 \%$, specificity $85.8 \%$ ) and 20 (sensibility $73.7 \%$, specificity $71.8 \%$ ), and showed that de EAT-40 significantly differentiates across groups with and without eating disorder diagnosis.
\end{abstract}

Key Words: Eating Attitudes Test, eating disorders, psychometrics, validity, reliability. 


\section{Introducción}

Los tests psicológicos tienen gran importancia en la vida moderna; los procedimientos de medida se aplican en decisiones importantes tales como diagnósticos clínicos, clasificaciones escolares, contrataciones laborales, etc., por lo que tienen gran influencia sobre la vida de las personas. Dada esta relevancia, es fundamental contar con test científicos y conocer sus limitaciones para, con ello, dar un mejor tratamiento a los problemas de la medición psicológica.

En esta investigación interesa el problema de los trastornos de la conducta alimentaria (TCA). La prevalencia de este tipo de condiciones parece haber aumentado en los últimos años, principalmente en la población femenina y en las sociedades industrializadas, en las que estar delgado se relaciona estrechamente con el atractivo físico, la belleza, la aceptación y el logro (Salazar, 2008). La American Psychiatric Association (APA, 2000/2002) define los TCA como condiciones caracterizadas por alteraciones graves en este tipo de conducta que incluyen la anorexia nerviosa y la bulimia nerviosa. Una característica fundamental de ambos diagnósticos es la alteración de la percepción de la forma y el peso corporales. La anorexia se caracteriza por un miedo intenso a ganar peso, el rechazo a comer suficiente y una constante molestia sobre la propia imagen corporal. Por su parte, la bulimia se caracteriza por ingerir grandes cantidades de alimentos en discretos intervalos de tiempo, seguido por intentos de compensar la sobreingesta mediante el vómito, el uso de laxantes, dietas restrictivas o de ejercicio.

Tanto la anorexia como la bulimia son cuadros complejos, compuestos por múltiples factores que interactúan entre sí y generan modificaciones en prácticamente todas las áreas de la vida de la persona. Implican cambios en el pensamiento que inciden, además, en las emociones, ya que se generan ciertos comportamientos que repercuten en el entorno y se sufren en el organismo. Se tiene bien claro que algunas de las consecuencias generadas por la anorexia y la bulimia son irreversibles (Cervera, 1996; Harrison \& Cantor, 1997; Toro \& Vilardell, 1987) y estos trastornos amenazan hoy en día la salud mental y física de un alarmante número de mujeres. La American Psychiatric Association (2000/2002) estima que la prevalencia de la anorexia nerviosa durante la vida es, en las mujeres, de aproximadamente un $0,5 \%$ y que la prevalencia en hombres es aproximadamente una décima parte de la prevalencia en mujeres. En el caso de la bulimia nerviosa, la prevalencia entre las mujeres es aproximadamente del $1 \%$ al 3\%; entre los hombres la prevalencia es diez veces menor. Por tanto, la prevalencia de TCA específicos (anorexia, bulimia) está entre el 0,5\% y el 3\% de la población general. Por otra parte, entre el $4 \%$ y el $22 \%$ de las mujeres universitarias 
reportan estar relacionadas con un comportamiento anoréxico o bulímico (Harrison \& Cantor, 1997). Según Zeledón (2004) en el caso particular de la bulimia, diferentes investigadores consideran que estos cuadros de desorden alimenticio se encuentran muy cerca de definirse como epidemia social.

Dos estudios realizados en Costa Rica con estudiantes de colegios públicos y privados, de ambos géneros y del área metropolitana, muestran porcentajes significativos de presencia de estos trastornos que alcanzan el $20 \%$. Como lo muestran la investigación de Salazar (2008) con una muestra de 533 adolescentes y la de Rosabal (2005) con 369, el mayor el riesgo lo tienen las mujeres.

Considerando su presencia y gravedad se ve la necesidad de estudiar el fenómeno de los TCA en la población costarricense. Sin embargo, en nuestro país no contamos con instrumentos válidos y confiables para la investigación y el diagnóstico temprano de estos trastornos, que van en aumento en la población mundial y de los cuales no somos ajenos. De aquí el esfuerzo de la presente investigación por probar estadísticamente, en una muestra de jóvenes costarricenses, el comportamiento de uno de los instrumentos más usados para detectar el riesgo de desarrollar TCA, el Test de Actitudes hacia la Alimentación (Eating Attitudes Test: EAT- 40).

El EAT-40 fue diseñado por Garner y Garfinkel (1979) para evaluar las conductas y actitudes sobre la comida, el peso y el ejercicio relacionados con la anorexia. Consiste en un cuestionario autoadministrado de 40 ítems que admite seis posibles respuestas que van de "nunca" a "siempre" (existe una versión abreviada de 26 ítemes que posee una alta correlación con la original, EAT-26).

El EAT-40 se encuentra validado en España por Castro, Toro, Salamero y Guimerá (1991). La puntuación total del test distingue entre pacientes anoréxicas y población normal y entre bulímicas y población normal, pero no lo hace entre anoréxicas restrictivas y bulímicas. Estos autores compararon un grupo de mujeres diagnosticadas con anorexia y un grupo control. Observaron que su versión del test logró una discriminación adecuada entre ambos grupos (grupo anorexia $M=45.2$ (22.8), grupo control $M=18.1$ (10.4), ( $t$, por pares $=9.20$, con una $p<.0001)$. El punto de corte propuesto por los autores originales es de 30 (sensibilidad moderada de 100\% y especificidad de 93\%). La versión española (Castro et al., 1991) propone un punto de corte de 20 para alcanzar una sensibilidad de $91 \%$ y una especificidad de 69.2\%; estas diferencias se dan porque en la versión española se obtiene una puntuación media menor. La consistencia interna fue adecuada para la muestra total (alfa de Cronbach de .93) y para el grupo de anoréxicas (alfa de Cronbach de .92) y se obtuvo evidencia de validez concurrente con el Beck Depression Inventory $(r=.63)$. En el análisis factorial de los autores originales se obtuvieron siete componentes principales; en la versión española solo tres factores, a saber: "Dieta y 
preocupación por la comida", "Presión social percibida y malestar con la alimentación” y "Trastornos psicobiológicos". Estos tres factores explican el $41 \%$ de la varianza total. El primer factor "Dieta y preocupación por la comida" es el mejor establecido; los otros dos factores presentan menos homogeneidad con respecto a las agrupaciones factoriales en otros trabajos (Gutiérrez, Raich, Sánchez \& Deus, 2003). En la Tabla 1 se presentan los ítems del EAT-40 según el factor que miden; son los de la versión española validados cualitativamente por Salazar (2008) en población costarricense y tomados originalmente de Gutiérrez et al. (2003).

Para efectos de la presente investigación se trabajó con los 40 ítems de la versión española completa (Castro et al., 1991) para contar con mayor cantidad de reactivos, considerando la eliminación de estos al probarse con una muestra distinta.

Para iniciar los análisis de confiabilidad y la validez del EAT-40 se hizo una búsqueda de los principales y más recientes estudios realizados a dicho test, esto permitió definir con mayor claridad y certeza los análisis a realizar.

Desde su creación en 1979 (Garner \& Garfinkel, 1979), el EAT-40 ha sido estudiado y utilizado en muchos países tales como Estados Unidos (Garner, Olmsted, Bohr \& Garfinkel, 1982; Kanekoa, 2007; Mintz \& O’Halloran, 2000; Smead \& Richert, 1990), España (Castro et al., 1991; De Irala et al., 2008; Jáuregui et al., 2009; Merino, Pombo \& Godás, 2001; Peláez, Labrador \& Raich, 2007; Vázquez, García-Bóveda \& VázquezMorejón, 2007), Turquía (Akdevelioglu \& Gümüs, 2010; Buyukgoze-Kavas, 2007; Keskín, Engin \& Dulgerler, 2010), Portugal (Macedo et al., 2007; Pereira et al., 2008), México (Austin \& Smith, 2008), Italia (Preti et al., 2007) y Bulgaria (Boyadjieva \& Steinhausen, 1996). En todos estos contextos, la consistencia interna de la escala completa ha sido reiteradamente demostrada, alcanzando alfas de Cronbach en un rango de .71 (Preti et al., 2007) a .94 (Garner \& Garfinkel, 1979). Asimismo, se ha encontrado evidencia consistente de la estabilidad temporal de las puntuaciones obtenidas con el EAT-40 (Macedo et al., 2007; Savasir \& Erol, 1989, citado en Buyukgoze-Kavas, 2007; Soares, Macedo, Gomes, \& Azevedo, 2004, citado en Macedo et al., 2007).

Por otra parte, a pesar de que el estudio que dio origen a la escala reportó una estructura factorial de siete componentes (Garner \& Garfinkel, 1979), en la actualidad se ha obtenido evidencia transcultural de una estructura de tres factores, tanto en los Estados Unidos (Garner et al., 1982) como en otros países (Boyadjieva \& Steinhausen, 1996; Castro et al., 1991; Macedo et al., 2007; Pereira et al., 2008). Aunque la composición de dichos factores cambia de un estudio a otro, los componentes más observados han sido a) dieta, b) comportamientos bulímicos y c) presión social para comer. Otros estudios han encontrado estructuras de cinco (Merino et al., 2001) y seis factores (Smead \& Richert, 1990). De todos los estudios consultados, el 
Tabla 1.

Estructura factorial del Test de Actitudes hacia la Alimentación encontrado en Castro et al. (1991)

\begin{tabular}{|c|c|c|c|}
\hline \multirow{2}{*}{ Ítems } & \multicolumn{3}{|c|}{ Factor } \\
\hline & $\mathrm{I}^{\mathrm{a}}$ & $\mathrm{III}^{\mathrm{b}}$ & 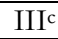 \\
\hline 1. Me gusta comer con otras personas & & & \\
\hline 2. Preparo comidas para otros, pero yo me las como & I & & \\
\hline 3. Me pongo nervioso/a cuando se acerca la hora de las comidas & I & & \\
\hline 4. Me da mucho miedo pesar demasiado & I & & \\
\hline 5. Procuro no comer, aunque tenga hambre & I & & \\
\hline 6. Me preocupo mucho por la comida & I & & \\
\hline $\begin{array}{l}\text { 7. A veces me "lleno" de comida sintiendo que soy incapaz de parar } \\
\text { de comer }\end{array}$ & & & \\
\hline 8. Corto mis alimentos en trozos pequeños & I & II & \\
\hline 9. Tengo en cuenta las calorías que tienen los alimentos que como & $\mathrm{I}$ & & \\
\hline $\begin{array}{l}\text { 10. Evito, especialmente, comer alimentos con muchos hidratos de } \\
\text { carbono (por ejemplo: pan, arroz, papas, etc.) }\end{array}$ & I & & \\
\hline 11. Me siento lleno/a después de las comidas & & & \\
\hline 12. Noto que los demás preferirían que yo no comiera más & & II & \\
\hline 13. Vomito después de haber comido & & & III \\
\hline 14. Me siento muy culpable después de comer & I & & \\
\hline 15. Me preocupa el deseo de estar más delgado/a & I & & \\
\hline 16. Hago mucho ejercicio para quemar calorías & I & & \\
\hline 17. Me peso varias veces al día & I & & \\
\hline 18. Me gusta que la ropa me quede ajustada & & & \\
\hline 19. Disfruto comiendo carne & & & III \\
\hline 20. Me levanto pronto por las mañanas & I & & \\
\hline 21. Cada día como los mismos alimentos & I & & \\
\hline 22. Pienso en quemar calorías cuando hago ejercicio & I & & \\
\hline 23. Tengo la menstruación regular (varones pasan a la siguiente) & & & \\
\hline 24. Los demás piensan que estoy demasiado delgado/a & & II & \\
\hline 25. Me preocupa la idea de tener grasa en el cuerpo & I & & \\
\hline 26. Tardo en comer más que las otras personas & & II & \\
\hline 27. Disfruto comiendo en restaurantes & I & & \\
\hline 28. Tomo laxantes (purgantes) & & & III \\
\hline 29. Procuro no comer alimentos con azúcar & I & & \\
\hline 30. Como alimentos de régimen (dieta) & I & & \\
\hline 31. Siento que los alimentos controlan mi vida & I & & \\
\hline 32. Me controlo en las comidas & I & & \\
\hline 33. Noto que los demás me presionan para que coma & & II & \\
\hline 34. Paso demasiado tiempo pensando y ocupándome de la comida & I & & \\
\hline 35. Tengo estreñimiento & & & III \\
\hline 36. Me siento incómodo/a después de comer dulces & I & & \\
\hline 37. Me comprometo a hacer régimen (dieta) & I & & \\
\hline 38. Me gusta sentir el estómago vacío & I & & \\
\hline 39. Disfruto probando comidas nuevas y sabrosas & I & & \\
\hline 40. Tengo ganas de vomitar después de las comidas & & & III \\
\hline
\end{tabular}

\footnotetext{
a Dieta y preocupación por la comida

b Presión social percibida y malestar con la alimentación

c Trastornos psicobiológicos
} 
único que incluyó la consistencia interna de cada factor dentro de sus resultados fue el de Pereira et al. (2008), quienes obtuvieron alfas de Cronbach entre .670 y .839 en cada uno de los tres factores observados.

Algunos estudios han obtenido validez de criterio del EAT-40 al encontrar correlaciones entre las puntuaciones en este instrumento y variables tales como insatisfacción con la imagen corporal (Jáuregui et al., 2009; Kanekoa, 2007), depresión (Buyukgoze-Kavas, 2007; Castro et al., 1991), perfeccionismo (Macedo et al., 2007), discrepancia con el peso deseado (Castro et al., 1991) e incluso con otras estimaciones de la bulimia nerviosa (Vázquez et al., 2007).

Asimismo, se ha observado que las puntuaciones en el EAT-40 difieren entre personas con un TCA diagnosticado y personas sin TCA en contextos tales como México (Alvarez-Rayón et al., 2002, citado en Austin \& Smith, 2008), Bulgaria (Boyadjieva \& Steinhausen, 1996), España (Castro et al., 1991) y Estados Unidos (Mintz \& O’Halloran, 2000).

Otro grupo de investigaciones se ha avocado a examinar la validez del EAT-40 en cuanto a su capacidad para diferenciar entre personas que tienen o no un TCA. En estos casos, se han comparado las puntuaciones del EAT40 con un diagnóstico clínico previo o con los resultados de una entrevista clínica posterior. Dichas investigaciones se interesaron en determinar otro tipo de propiedades del EAT-40 tales como su nivel de sensibilidad (porcentaje de verdaderos positivos de entre el total de personas con TCA) y de especificidad (porcentaje de verdaderos negativos de entre el total de personas sin TCA). Además de los datos de validez aportados, este tipo de análisis arrojó información acerca de cuál puede ser un punto de corte adecuado para que mediante el EAT-40 se distinga el máximo de personas con riesgo de desarrollar un TCA sin caer en un alto nivel de falsos positivos. Acorde con lo planteado por Garner y Garfinkel en el estudio original (1979), el puntaje de corte más utilizado en las investigaciones consultadas fue el de 30, con porcentajes de sensibilidad y especificidad que van desde cifras moderadas (52.7\% y 93.8\% respectivamente, De Irala et al., 2008) hasta estadísticas muy prometedoras (77\% y 95\% respectivamente, Mintz \& O'Halloran, 2000). Adicionalmente, De Irala et al. (2008) han recomendado utilizar el puntaje de corte de 21 (sensibilidad 73.3\%, especificidad 85.1\%) mientras que Castro et al. (1991) recomiendan utilizar un corte de 20 (sensibilidad $91 \%$, especificidad 69.2\%). 


\section{Método}

Participantes

Los criterios de selección del grupo se basaron en estudios anteriores tanto a nivel nacional como internacional (documentados en Salazar, 2008), donde la mayor prevalencia del TCA es en población joven, femenina, universitaria, de estratos altos, que practican ciertos deportes y habitan en las zonas industrializadas. La muestra quedó compuesta por un total de 569 mujeres del área metropolitana de San José, Costa Rica (ver Tabla 2). Las participantes del estudio se seleccionaron de diferentes grupos con el fin de obtener diversos estados de TCA; a saber: "Muestra normal" (de diversos niveles socioeconómicos, no internadas y no diagnosticadas con TCA), "Muestra con riesgo" (estudiantes de academias de ballet y jóvenes que pertenecen a foros en Internet pro anorexia, pero no diagnosticadas con TCA) y "Muestra diagnosticada con TCA" (diagnosticadas con TCA por psicólogos o psiquiatras del sistema de salud costarricense e internadas en hospitales).

Tabla 2.

Composición de la muestra total del estudio

\begin{tabular}{lccc}
\hline Grupo & $n$ & $\%$ & Nivel socieconómico \\
\hline Muestra normal & 511 & 89.8 & - \\
$\quad$ Colegio diurno público y & 212 & 37.2 & Medio-amplio \\
$\quad$ privado & 119 & 20.9 & Medio-bajo \\
Colegio nocturno & 120 & 21.1 & Medio-amplio y medio \\
Universidad & 60 & 10.5 & alto \\
Colegio privado & 28 & 4.9 & Medio-alto \\
Muestra con riesgo & 26 & 4.6 & - \\
Academia de ballet & 2 & 0.4 & - \\
Pro anorexia & 30 & 5.3 & - \\
Muestra con TCA diagnosticado & 5 & 0.9 & - \\
Hospital Psiquiátrico & 8 & 1.4 & - \\
Hospital Calderón Guardia & 17 & 3.0 & \\
Hospital Nacional de Niños & 569 & 100 & \\
\hline Total & & &
\end{tabular}

El grupo muestral fue suficiente para la capacidad operativa de la recolección de datos, para realizar los análisis paramétricos necesarios y para cumplir con los objetivos del estudio. La muestra fue de tipo no probabilística, intencional (Kerlinger \& Lee, 2000/2002), es decir, los instrumentos se aplicaron a la totalidad de casos con TCA encontrados 
mientras que, por otra parte, las demás participantes estuvieron disponibles en sus aulas, academias de ballet o por la Web y no fueron seleccionados al azar. En los dos casos de pro anorexia se realizaron entrevistas a través de Skype. Asimismo, las participantes fueron todas voluntarias y completaron un consentimiento informado. En el caso de las menores de edad, este fue avalado por sus padres o encargados.

La obtención de casos con diagnóstico de anorexia o bulimia resultó difícil, ya que correspondían a pacientes de los servicios de Psiquiatría y Psicología de hospitales y el número de personas que llega a estos centros no es muy alto. Por un periodo de 3 meses se analizaron los casos con anorexia y bulimia que acudían a los hospitales Nacional Psiquiátrico, Calderón Guardia y el Departamento de Atención al Adolescente del Hospital Nacional de Niños; en ese tiempo se logró obtener 30 casos debidamente identificados y diagnosticados por psiquiatras o psicólogos. Aquí nos dimos cuenta que el porcentaje que llega a estos Centros es bajo, ya que son importantes centros de salud del país en cuanto a cantidad de población que atienden. Además, una de las características de los TCA es la de mantener la sintomatología en secreto, por eso se considera que hay más casos de los documentados. Esto se pudo apreciar al observar que son muchas las mujeres jóvenes que pertenecen a grupos pro anorexia en la Web. Sin embargo, por ese medio no se podía comprobar el diagnóstico profesional aunque el estado físico lo sugiriera (en el caso de las anoréxicas). El número reducido de casos diagnosticados (4 de bulimia y 15 de anorexia) no afectó al estudio, ya que el principal objetivo de este consistió en verificar el nivel de eficiencia del instrumento, es decir, evaluar si esta herramienta era capaz de identificar la presencia del TCA en pacientes diagnosticadas.

Con respecto a la edad de las mujeres participantes, el rango tiene un valor mínimo de 10 años y uno máximo de 39 años; edades tan distantes se debe a que hubo población universitaria involucrada, de colegio nocturno y estudiantes de academias de ballet. El promedio de edad fue de 17.66 años y hubo un importante grado de dispersión $(D E=6.65)$. El rango de edad de la muestra de casos diagnosticados es de 13 a 23 años $(n=19, M=16.58, D E$ $=2.45$ ), mientras que el resto de la muestra se mantuvo dentro de estas edades a excepción de 30 personas (solo 4 de ellas superan los 29 años). La edad de la muestra sin diagnóstico de TCA no difirió significativamente de la muestra con diagnóstico $\left(n=533, M=17.31, D E=3.18, t_{(550)}=.324\right)$, por lo que ambos grupos son comparables en cuanto a edad.

Se consideró el colegio de procedencia, ya que es una variable, entre otras, que representa el nivel socioeconómico. De acuerdo con investigaciones consultadas (Salazar, 2008), las personas con mayores ingresos son más propensas a presentar TCA, por lo que fue importante la inclusión de dicha característica. El 56\% del grupo de estudio proviene de colegios públicos y el $44 \%$ de colegios privados. 


\section{Procedimientos}

Una vez adaptada la prueba al lenguaje y habiendo modificado algunos ítems (ver prueba piloto en Salazar, 2008) se solicitó la autorización de las personas responsables de los colegios, academias y hospitales para aplicar la prueba. El cuestionario que incluía el EAT-40 se entregó y recogió en un sobre manila. En el caso de los colegios se aplicó en las aulas y cada participante contestó de manera individual, en el resto de los casos la aplicación fue individual. Las instrucciones fueron comentadas en voz alta (las mismas para todas las participantes) y se atendieron las dudas que surgieron mientras completaban la información.

\section{Estrategia de análisis}

El análisis de la información se llevó a cabo con la utilización del Paquete Estadístico para las Ciencias Sociales (SPSS, versión 17) y el AMOS (Analysis of Moment Structures, versión 16). En una primera parte se procedió con un análisis factorial confirmatorio, para luego medir la confiabilidad interna de cada subescala; seguidamente se realizó una clasificación descriptiva del diagnóstico, y luego se corroboraron las diferencias de los grupos según el trastorno de acuerdo con el análisis de validación de criterio.

\section{Análisis factorial}

\section{Resultados}

Con el fin de detectar la estructura interna del constructo en el EAT-40, se llevó a cabo tanto el análisis factorial exploratorio (AFE) como el análisis factorial confirmatorio (AFC). Si bien en este caso se tiene una base teórica para poder definir a priori los factores que conformarán la estructura de la herramienta, la combinación de análisis exploratorio y confirmatorio es comúnmente recomendada para brindar una validez estructural más consistente (Wegener \& Fabrigar, 2000). Para el AFE se decidió tomar un $20 \%$ de la muestra total (124 participantes) mientras que para el AFC se utilizaron los datos de todas las participantes del estudio (569 participantes).

\section{Análisis factorial exploratorio}

La prueba de adecuidad del modelo factorial (KMO: Kaiser-MeyerOlkin) resultó relativamente alta (.725), en la prueba de esfericidad de Bartlett se obtuvo un valor significativo $(p<.001)$ y el estudio de 
comunalidades mostró valores (extracción) mayores o muy cercanos a .7 en la gran mayoría de los ítems. Estos tres indicadores permitieron corroborar la justa utilización del análisis factorial. Dicho análisis se realizó mediante la "Varianza total explicada" y el "Gráfico de sedimentación". En el primer caso, el criterio para determinar el número total de factores fue que cada uno de los componentes explicara más de un $10 \%$ de la varianza. Al utilizar la varianza total explicada como criterio en el AFE, se encontró que el primer factor explicó el $24.79 \%$ de la varianza mientras que de los factores restantes el mayor explicó apenas un $6.38 \%$. A partir de este criterio, pareciera que la prueba es explicada mediante un único factor. Sin embargo, el criterio de la varianza explicada se debe utilizar en conjunto con el "gráfico de sedimentación" para visualizar posibles componentes que podrían ser tomados en cuenta. El análisis de la gráfica de sedimentación producto del AFE arrojó tres factores que comprenden el total de ítems de la prueba.

Dado que la "Varianza total explicada" y el "Gráfico de sedimentación" llegan a resultados diferentes (el primero da indicios de que se debe optar por un único componente mientras que la segunda técnica muestra tres factores), se procedió a efectuar el AFC, con el cual se corroboró la estructura factorial de la prueba.

\section{Análisis factorial confirmatorio}

Se hicieron dos AFC, uno utilizando un único factor que explica los ítems que normalmente se utilizan para llevar a cabo la validación y otro que utiliza solamente los ítemes comprendidos en los tres factores de la versión española de la escala, a saber: "Dieta y preocupación por la comida", "Presión social percibida y malestar con la alimentación" y "Trastornos psicobiológicos" (ver Tabla 1). Para determinar cuál de los dos modelos ajustaba mejor, se compararon los índices y el valor de la Chi-cuadrado sobre los grados de estos dos modelos. Vale señalar que en estos análisis no se tomaron en cuenta los ítems número 1, 7, 11, 18 y 23 porque estos no se consideraron en la clasificación de los ítemes en tres factores de la versión española y este es el modelo teórico que se siguió en estos análisis.

Modelo con todos los ítems: para conocer el ajuste del modelo confirmatorio para un solo factor se tomaron en cuenta varios índices y el criterio del valor de la Chi-cuadrado entre los grados de libertad del modelo. Se pudo ver que en general el modelo con un factor ajusta moderadamente bien. Se considera que un modelo es bueno si el valor de la Chi-cuadrada está entre 1 a 3 grados de libertad, y aceptable entre 3 a 5; en el presente caso el valor fue de 4.65, por lo que el modelo ajusta aceptablemente. Otros de los criterios que utiliza el AMOS consisten en el "Índice de ajuste relativo (RIF)", el "Índice de ajuste incremental (IFI)", el "Coeficiente de TuckerLewis", el "Índice de ajuste comparativo (IAC)", entre otros. Entre más 
cercano a 1 estén los valores del coeficiente y de los índices de cada uno de estos criterios se dice que el ajuste del modelo es bueno. Asimismo, valores por debajo de .6 indican que el modelo con el número de factores presentes no es tan adecuado. Para el presente caso, todos los índices y coeficiente son mayores a .6, siendo .63 el menor entre ellos; de igual forma, se encontró que en promedio los valores son cercanos a .7, lo cual indica un ajuste aceptable. Por lo tanto, de acuerdo con los resultados indicados, el modelo de un solo factor posee un buen ajuste.

Modelo con los tres factores: en estudios anteriores, el análisis confirmatorio se realizó con ítems que podían cargar en más de un factor (es el caso de los ítems 3, 5, 8, 29, y 34). Generalmente, cuando se construye cualquier tipo de pregunta de investigación o de evaluación, se recomienda que el ítem sea totalmente unidireccional, esto es, que la pregunta no tenga ningún tipo de ambigüedad que pueda confundir a quien la responde. Por lo tanto, se decidió incluir los ítems que teóricamente cargan en dos factores, únicamente en un factor, y el criterio para elegir el factor fue analizando en dónde había una mayor carga factorial (ver distribución en Tabla 1).

Para comprobar si el modelo ajustaba bien con la composición factorial de 3 factores, se verificaron los mismos criterios mencionados para el modelo de un solo factor y se encontró que el modelo ajusta relativamente bien: el valor de Chi-cuadrado entre los grados de libertad es de 4.12 y además todos los índices son superiores a .6, siendo el mínimo .67 y el promedio .73. Por lo tanto, se verifica que también el modelo factorial confirmatorio con tres factores posee un ajuste aceptable.

Finalmente, fue importante ver cuál de los dos modelos ajusta mejor con tal de hacer la validación interna de la escala. Para esto se compararon los valores de la Chi-cuadrado y de los índices para cada modelo. De acuerdo con los resultados se concluye que el modelo confirmatorio de tres factores es superior al de un solo factor: el valor de la Chi-cuadrada entre los grados de libertad es menor para el modelo de tres factores $(4.12<4.65)$; también, para cada uno de los índices y del coeficiente, los valores del modelo con tres factores se ajustan mejor debido a que están más cercanos al 1. La composición de los tres factores resultantes del análisis factorial resultó como la teoría lo sugiere. Por lo tanto, para los análisis de validez interna de la prueba, se prosiguió mediante esta estructura factorial.

\section{Consistencia interna}

La escala completa, con sus 40 ítems, obtuvo un alfa de Cronbach de .889 , mientras que, al explorar la consistencia de la versión de tres factores de Castro et al. (1991) en nuestra muestra se obtuvo un alfa de Cronbach de .899. Por otra parte, con base en la estructura factorial mencionada anteriormente, se llevó a cabo un análisis de consistencia interna para cada 
uno de los tres componentes. Primero se analizó la significancia de los ítems en la regresión de los pesos, luego se determinó cuáles reactivos poseían un direccionamiento inverso, y finalmente se hizo el estudio de consistencia interna para cada una de las subescalas.

Considerando la significancia de los ítems, en el factor de "Dieta y preocupación por la comida", dos de los ítems no resultaron significativos (el 2 y 20, el valor del coeficiente es de $p>$.05), por consiguiente fueron eliminados del modelo a la hora de llevar a cabo el análisis de confiabilidad. En los otros dos factores, a saber: "Presión social percibida y malestar con la alimentación" y "Trastornos psicobiológicos", todos los ítems presentaron valores de significancia $(p<.05)$, por lo cual en estas subescalas la estructura no se modificó.

Posteriormente, se verificó cuáles ítems poseían un direccionamiento negativo, con el fin invertir los valores de las respuestas. Solamente en el factor "Dieta y preocupación por la comida" los ítems 27 y 39 resultaron negativos en sus estimaciones estandarizadas, por lo que se les cambió el direccionamiento a sus respuestas. Entonces, mediante los diagnósticos anteriores, se procedió a la respectiva eliminación de los ítems y a la inversión de las respuestas, y finalmente se realizó el análisis de confiabilidad interna, donde se calculó el alfa de Cronbach y las correlaciones punto biserial para cada uno de los tres factores puestos en razón.

El factor "Dieta y preocupación por la comida" mostró un alto grado de confiabilidad, pues presentó un alfa de Cronbach de .92 (la confiabilidad límite es de .8). También se verificó, a nivel de los ítems, si estos poseían suficiente calidad técnica para discriminar adecuadamente a los sujetos. Para esto se utilizó la correlación punto biserial, la cual se considera aceptable si los reactivos poseen valores superiores a .3; de lo contrario, se sugiere eliminar el ítem, ya que este no estaría aportando nada al análisis. Se encontró que los ítems 21 y 27 no discriminaron, por lo tanto estos se eliminan del análisis, lo cual, a su vez, produjo un valor de alfa de Cronbach más alto al volver a realizar el análisis: aumentó de .923 a .927.

El factor "Presión social percibida y malestar con la alimentación" obtuvo un índice de confiabilidad bajo (alfa de Cronbach de .46), por consiguiente, este factor no aportó ningún tipo de fidelidad a la prueba. Se analizó si, al eliminar algún ítem, la confiabilidad aumentaba de forma suficiente para considerarlo como adecuado, pero los resultados mostraron que con la eliminación de alguno o varios de los ítems el factor no aportaba mayor diferencia al valor del alfa de Cronbach, por lo que este factor podría perfectamente ser eliminado.

Para el factor "Trastornos psicobiológicos" el alfa de Cronbach fue insuficiente $(\alpha=.61)$. Se analizó si esto fue debido a algún ítem que no discriminara bien, pero en ninguno de los casos el alfa de Cronbach aumentó 
de forma significativa para llegar al criterio de .8. Por tanto, se concluyó que el tercer factor no se puede considerar confiable.

\section{Discriminación}

Una vez analizada la estructura factorial y la consistencia interna de la prueba se verificó si el instrumento tiene la suficiente validez para predecir cuáles sujetos pueden considerarse en riesgo de TCA y cuáles no. Para esto se consideró el puntaje de riesgo alimenticio diseñado por los autores originales de la escala (Garner \& Garfinkel, 1979) quienes utilizaron un punto de corte de 30 , es decir, si una persona presenta 30 puntos o menos no se le consideraba en riesgo de TCA, pero si se posee un puntaje de 31 o más, entonces se puede decir que está propenso a poseer un TCA. En la presente investigación, para el caso de las "Diagnosticados”, el 63\% de la muestra diagnosticada presentan potenciales TCA según sus puntuaciones en el EAT-40. Aunque sea una muestra bastante pequeña, por lo menos en términos de la distribución a nivel del puntaje, los resultados evidencian lo que se esperaba.

Por otra parte, en la "Muestra sin diagnóstico", la distribución de los datos tiende a aglomerarse principalmente en los puntajes más bajos (aunque siempre hay presencia de puntajes altos, como es de esperar). En esta muestra más del $85 \%$ posee valores de puntaje inferiores a los 31 , lo cual es un resultado deseable, ya que se esperaba que la mayor proporción de esta muestra no estuviera en riesgo de TCA, ya que las mujeres fueron elegidas sin tener un diagnóstico previo.

Considerando el punto de corte que caracteriza mejor el potencial del TCA se realizaron también los cálculos con un corte de 20 , siguiendo el criterio de Castro et al. (1991) y se analizó de qué forma este hecho podría influir en la clasificación de las mujeres para la presente prueba (ver Tabla 3).

De acuerdo con los resultados de la Tabla 3, se puede observar, primeramente, que a nivel del punto de corte de 30, se obtienen los resultados esperados. Ahora, si se hace un balance entre los puntos de corte de 20 y 30, pareciera que el menor es el que aporta mejores propiedades, porque se gana mayor detectabilidad a nivel de potenciales riesgos alimenticios. Para próximas aplicaciones, se podría manejar el criterio de 20 , ya que, además, el total de ítems sería reducido de acuerdo con los análisis de confiabilidad vistos anteriormente 
Tabla 3.

Índices de discriminación según puntaje de corte utilizado en el Test de Actitudes hacia la Alimentación

\begin{tabular}{|c|c|c|c|c|}
\hline & \multicolumn{2}{|c|}{ Con diagnóstico } & \multicolumn{2}{|c|}{ Sin diagnóstico } \\
\hline $\begin{array}{l}\text { Puntaje } \\
\text { de corte }\end{array}$ & $\begin{array}{l}\text { Verdaderos } \\
\text { positivos }\end{array}$ & $\begin{array}{c}\text { Falsos } \\
\text { negativos }\end{array}$ & $\begin{array}{c}\text { Verdaderos } \\
\text { negativos }\end{array}$ & $\begin{array}{c}\text { Falsos } \\
\text { positivos }\end{array}$ \\
\hline 20 & $14(73.7 \%)$ & $5(26.3 \%)$ & $385(71.8 \%)$ & $151(28.2 \%)$ \\
\hline 30 & $12(63.2 \%)$ & $7(36.8 \%)$ & $460(85.8 \%)$ & $76(14.2 \%)$ \\
\hline
\end{tabular}

Validez de criterio

A partir de los puntajes que se generaron del EAT- 40 se realizaron diferentes pruebas de hipótesis para observar si la prueba encontró diferencias de predictibilidad considerando las siguientes variables: tipo de grupo (Colegio, Hospital, Ballet/por anorexia y Universidad), diagnóstico, edad y colegio de procedencia. Este fue un criterio definitivo para determinar la validez de pronóstico de la prueba dependiendo del tipo de grupo a comparar. El resultado que más interesó fue detectar entre personas con riesgos alimenticios y aquellas que no lo poseen.

Institución. Se agruparon las submuestras del estudio de la siguiente manera, tomando en cuenta que los tamaños de dichas submuestran no influyeran en los análisis: las participantes de colegios diurnos, nocturnos y colegio de clase alta se agruparon en un conjunto general llamado "Colegio"; las pacientes de los hospitales Psiquiátrico, Calderón Guardia y Nacional de Niños se fusionaron en la categoría "Hospital"; las participantes reclutadas en academias de Ballet y comunidades Web pro anorexia se agruparon en la categoría "Ballet e Internet"; mientras que las participantes provenientes de universidades se conservaron bajo la categoría "Universidad".

Con base en estos nuevos grupos, se analizó si existían diferencias entre ellos a nivel del puntaje en el EAT-40. Mediante el análisis de variancia (ANOVA) con estos grupos se observó que la hipótesis de igualdad de medias no se debe aceptar. En efecto, la prueba resultó ser significativa $\left(F_{3,}\right.$ 564) $=33.976, p<.001)$, por lo tanto se rechazó la hipótesis nula de igualdad de medias a nivel de la puntuación del EAT-40.

Para determinar entre qué grupos específicos hubo diferencias significativas en el puntaje del EAT-40, se llevó a cabo el análisis de comparación múltiple (Tukey). En este se pudo observar que, acorde con lo esperado, no hubo diferencias significativas entre los grupos "Colegio" $(n=$ 391, $M=17.38, D E=12.63)$, "Universidad" $(n=120, M=15.64, D E=$ 12.80) y "Ballet e Internet" $(n=28, M=19.36, D E=19.78)$, pero sí al comparar cada uno de estos grupos contra el grupo "Hospital" $(n=29, M=$ $43.72, D E=25.42, p<.001)$. En la Figura 1 se puede observar la relación 
ascendente que se encontró entre los tipos de grupos. El grupo "Colegio" obtuvo los menores puntajes en el EAT-40, mientras que el grupo "Hospitales" obtuvo las mayores puntuaciones.

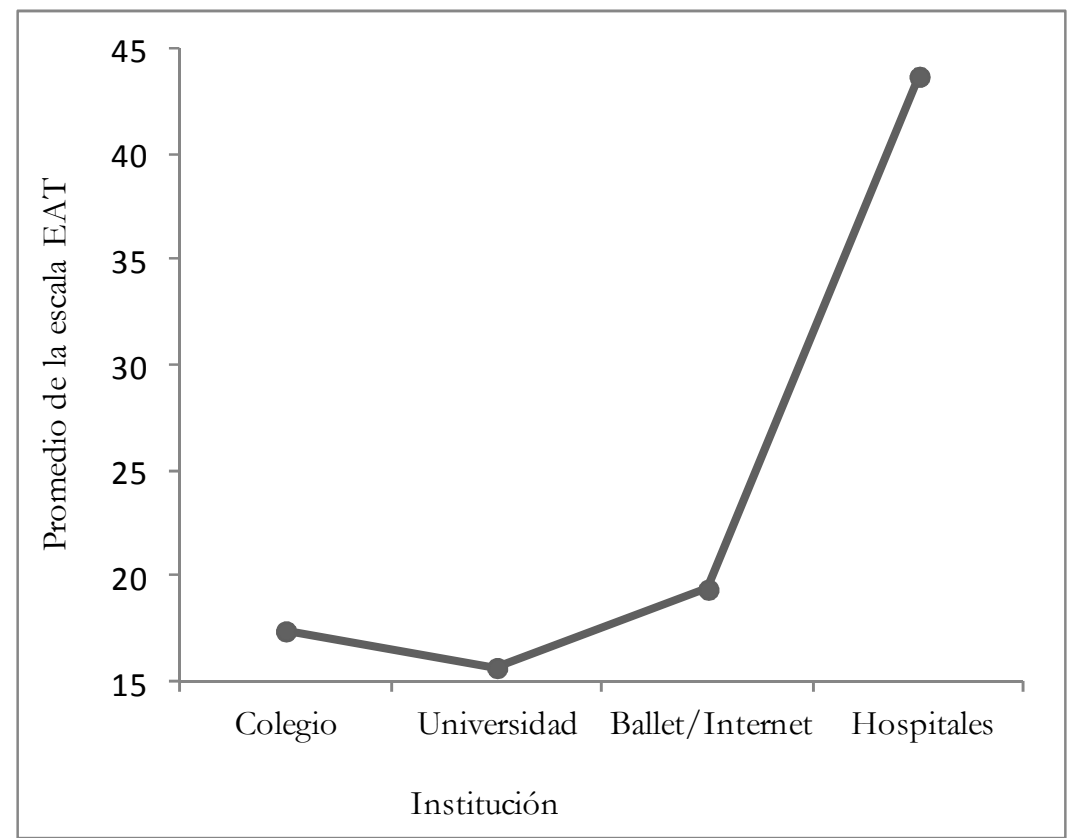

Figura 1. Puntuaciones promedio en el EAT-40 de participantes provenientes de distintas instituciones.

Diagnóstico. La prueba de comparación múltiple de ANOVA para observar si existieron diferencias en el puntaje del EAT-40 entre participantes diagnosticadas con anorexia, bulimia o no diagnosticadas demostró que la hipótesis de igualdad de medias se rechaza. En efecto, la prueba resultó ser significativa $\left(F_{(2,552)}=45.555, p<.001\right)$, por lo tanto no se acepta la hipótesis nula de igualdad de medias. La prueba Post-Hoc de Tukey permitió visualizar a nivel de qué grupos se encontraron las diferencias. Se encontraron diferencias en las puntuaciones en el EAT-40 entre las participantes diagnosticadas con bulimia $(n=4, \mathrm{M}=67.50, \mathrm{DE}=$ 28.05) y aquellas diagnosticadas con anorexia $(n=15, \mathrm{M}=42.53, \mathrm{DE}=$ $26.08, p<.01$ ), entre las primeras y las personas sin diagnóstico ( $\mathrm{n}=536, \mathrm{M}$ $=17.64, D E=13.75, p<.001)$ y entre las personas diagnosticadas con anorexia y las personas sin diagnóstico $(p<.001)$. Asimismo, hubo diferencias significativas entre las personas diagnosticadas con TCA (bulimia o anorexia) $(n=19, M=47.79, D E=27.74)$ al compararlas contra las personas sin diagnóstico $\left(t_{(18.315)}=4.717, p<.001\right)$. Este último resultado es 
quizá el más importante del estudio, ya que es el que permite verificar que la herramienta es buena discriminando entre las personas con y sin diagnóstico de TCA. En la Figura 2 se puede observar los diferentes promedios del puntaje del EAT-40 entre los tres diferentes grupos de diagnosticadas. Nótese que, en promedio, las personas con diagnóstico poseen un puntaje mayor a 40, mientras que el grupo sin ningún diagnóstico posee en promedio inferior a 20 puntos.

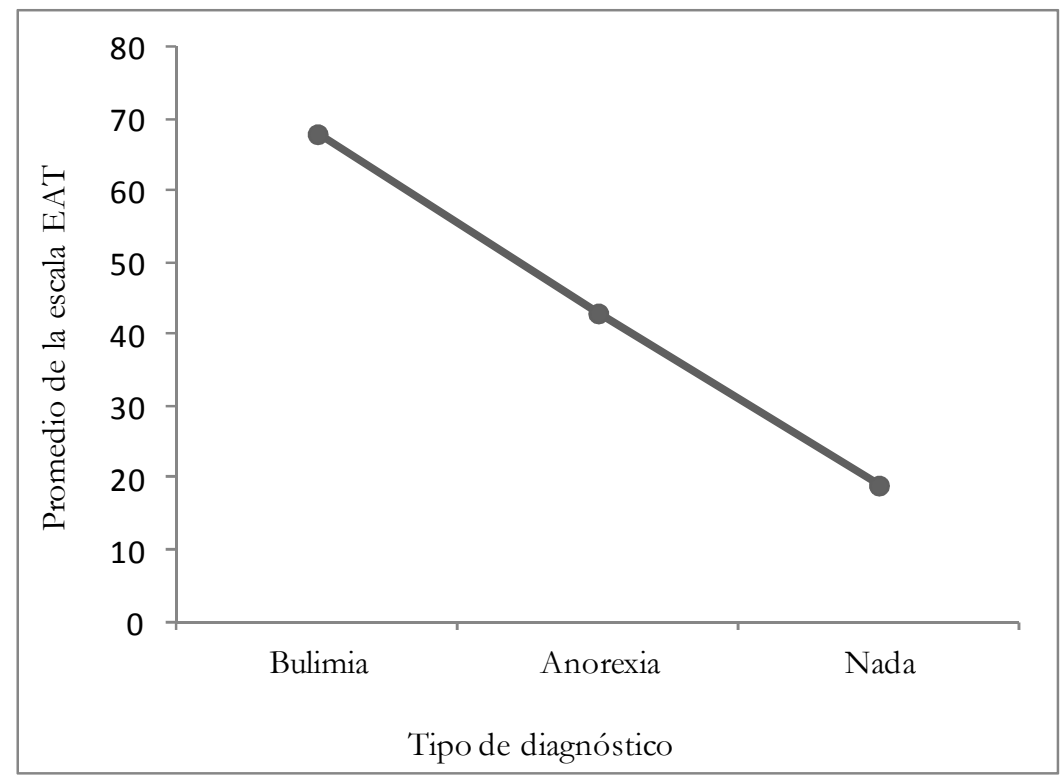

Figura 2. Puntuaciones promedio en el EAT-40 de participantes con distinto diagnóstico.

Edad. Las edades fueron agrupadas en tres categorías, considerando: "Adolescencia" (de 10 a 17 años), "Adultez prematura" (18 a 25 años) y "Adultez media" (26 a 39 años). De acuerdo con el análisis de variancia, se observó que la hipótesis de igualdad de medias no se debe aceptar. La prueba resultó ser significativa $\left(F_{(2,560)}=8.642, p<.001\right)$, por lo tanto se rechaza la hipótesis nula de igualdad de medias a nivel de la edad en la escala del EAT40. Como la prueba resultó significativa, se llevó a cabo un análisis Post Hoc para ver entre qué grupos específicos existían diferencias significativas. La adultez media $(n=24, M=17.13, D E=9.97)$ no se diferenció de la adultez prematura $(n=84, M=24.86, D E=19.84, \mathrm{p}=.070)$ ni de la adolescencia $(n$ $=461, M=17.46, D E=14.28, p=994)$, pero esta última sí se diferenció de la adultez prematura $(\phi<.001)$, la cual obtuvo las mayores puntuaciones (véase Figura 3). 


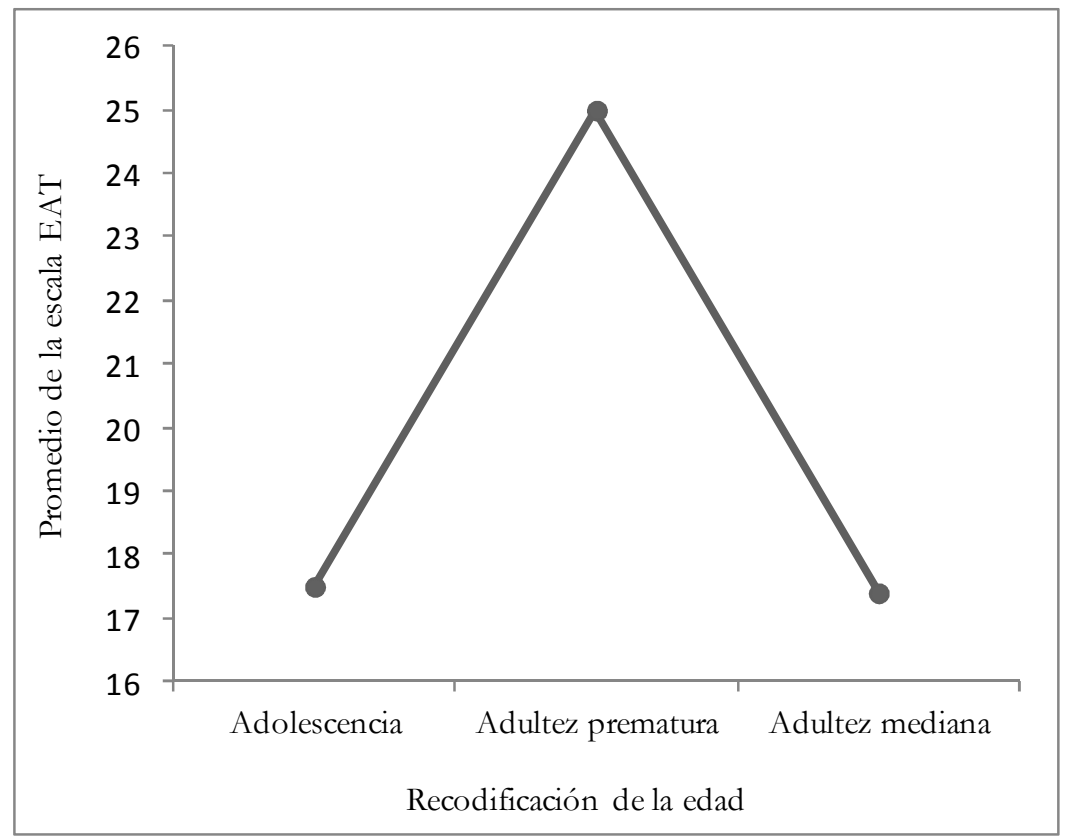

Figura 3. Puntuaciones promedio en el EAT-40 de participantes de distintos grupos etarios.

Colegio de procedencia. Se encontró que las personas de colegio público (n $=303, M=20.31, D E=16.39)$ puntuaron más alto que las de colegio privado $\left(n=238, M=15.66, D E=12,47, t_{(539)}=3.744, p<.001\right)$ en el EAT-40, lo cual va en contra de lo esperado. Se hipotetizaba que las personas con mayores ingresos, estudiantes en los colegios privados, presentarían puntuaciones mayores en el EAT-40 (Salazar, 2008).

\section{Conclusiones}

En esta investigación se exploró la confiabilidad y validez del EAT-40 en una muestra de 569 mujeres costarricenses que incluyó una submuestra normal de control (estudiantes de colegio y universidad no internadas ni diagnosticadas con TCA, $n=511$ ), una muestra de personas en riesgo de desarrollar un TCA (estudiantes de academias de ballet y jóvenes que pertenecen a foros pro anorexia en Internet, $n=28$ ) y una muestra clínica (mujeres diagnosticadas con bulimia o anorexia, $n=30$ ). 
La determinación de la validez del EAT-40 en mujeres costarricenses por medio del análisis factorial exploratorio nos demostró que el criterio de la variancia explicada reporta un solo factor. Sin embargo, el gráfico de sedimentación revela que el criterio del codo sugiere un modelo de tres factores. Este resultado se confirma con los dos tipos de análisis de factores confirmatorio: con un solo factor y considerando los tres factores que la teoría siguiere ("Dieta y preocupación por la comida", "Presión social percibida y malestar con la alimentación" y "Trastornos psicobiológicos"), donde se demuestra que tres factores ajustan mejor que un único factor, acorde con la literatura consultada.

La consistencia interna de la escala completa, con sus 40 ítems, demostró ser muy adecuada $(\alpha=.889)$, así como la consistencia obtenida al utilizar solamente los 35 ítems incluidos en los tres factores propuestos por Castro et al. (1991) $(\alpha=.899)$. Considerando la confiabilidad del EAT-40 para cada uno de los factores o subescalas, el factor de "Dieta y preocupación por la comida" (con 25 ítems), presentó altos niveles de confiabilidad (alfa de Cronbach .923; este aumentó a .927 sin los ítems 21 y 27). Con respecto al segundo ("Presión social percibida y malestar con la alimentación", de 5 ítems) y el tercer factor ("Trastornos psicobiológicos", de 5 ítems), no poseen niveles de confiabilidad aceptables (alfas de Cronbach de .46 y .61, respectivamente) y estos no se incrementan al eliminar ítems. Ante ello, se debe evitar llevar a cabo análisis a partir de estos factores, siendo lo recomendable utilizar la escala completa o los ítems de los tres factores juntos.

Con respecto a la capacidad del EAT-40 para predecir el riesgo de TCA, la herramienta funciona correctamente para diferenciar entre personas con riesgo alimenticio y las que no lo poseen. En la muestra costarricense, el puntaje de corte original de 30 en el EAT-40 demostró una moderada sensibilidad (63.2\%) y una muy buena especificidad (85.8\%). Por su parte, el puntaje de corte sugerido por Castro et al. (1991), establecido en 20, ofreció una mejor sensibilidad $(73.7 \%)$ pero a costa de bajar la especificidad del test $(71.8 \%)$.

Asimismo, se logró demostrar que las puntuaciones del EAT-40 diferenciaban claramente entre las personas con un diagnóstico de TCA y aquellas personas que no habían sido diagnosticadas. Al respecto, debemos señalar dos limitaciones del estudio. Por una parte, a pesar de los esfuerzos llevados a cabo, la muestra de jóvenes diagnosticadas con un TCA fue muy pequeña. Por otra parte, la muestra no diagnosticada no fue sometida a evaluación para asegurar que no padeciera un TCA. Ante esto, se debe tomar en cuenta que en este estudio se aportó evidencia de la capacidad del EAT40 para diferenciar entre personas diagnosticadas y no diagnosticadas con un TCA, mas no específicamente entre personas con y sin TCA. 
Con respecto a la agrupación por instituciones queda clara la diferencia significativa entre estos: poseen puntajes más altos las jóvenes diagnosticadas; posteriormente las no diagnosticas, donde obtienen puntajes más altos en el EAT-40 quienes practican ballet y pertenecen a foros pro anorexia, luego las universitarias y por último las estudiantes de colegio. A nivel de las edades, también se encontró diferencias significativas. Estas se refieren al grupo de edad intermedia "Adultez prematura" (18 a 25 años), ya que estas en promedio poseen una calificación mayor en la puntuación del EAT-40. Finalmente, el colegio de procedencia resultó ser un factor que influyó en el hecho de tener un posible riesgo alimenticio, pero en un sentido contrario al esperado: las jóvenes provenientes de colegios públicos obtuvieron mayores puntuaciones.

\section{Referencias}

Akdevelioglu, Y. \& Gümüs, H. (2010). Eating disorders and body image perception among university students. Pakistan Journal of Nutrition, 9(12), 1187-1191.

American Psychiatric Association. (2002). DSM-IV-TR. Manual diagnóstico y estadístico de los trastornos mentales (T. De Flores, J. Masana, E. Masana, J. Toro, J. Treserra \& C. Udina, Trad.). Barcelona, España: Masson, S. A. (Trabajo original publicado en 2000).

Austin, J. L. \& Smith, J. E. (2008). Thin ideal internalization in Mexican girls: a test of the sociocultural model of eating disorders. International Journal of Eating Disorders, 41(5), 448-457.

Boyadjieva, S. \& Steinhausen, H.-C. (1996). The Eating Attitudes Test and the Eating Disorders Inventory in four Bulgarian clinical and nonclinical samples. International Journal of Eating Disorders, 19(1), 93-98.

Buyukgoze-Kavas, A. (2007). Eating attitudes and depression in a Turkish sample. European Eating Disorders Review, 15, 305-310.

Castro, J., Toro, J., Salamero, M. \& Guimerá, E. (1991). The Eating Attitudes Test: Validation of the Spanish versión. Psychological Assessment, 7(2), 175-190.

Cervera, M. (1996). Riesgo y prevención de la anorexia y la bulimia. Barcelona, España: Martínez Roca.

De Irala, J., Cano-Prous, A., Lahortiga-Ramos, F., Gual-García, P., MartínezGonzález, M. A. \& Cervera-Enguix, S. (2008). Validación del cuestionario Eating Attitudes Test (EAT) como prueba de cribado de trastornos de la conducta alimentaria en la población general. Med Clin (Barc), 130(13), 487-491.

Garner, D. M. \& Garfinkel, P. E. (1979). The Eating Attitudes Test: an index of the symptomas of anorexia nervosa. Psychological Medicine, 9(22), 273-279.

Garner, D. M., Olmsted, M. P., Bohr, Y. \& Garfinkel, P. E. (1982). The Eating Attitudes Test: psychometric features and clinical correlates. Psychological Medicine, 12, 871-878.

Gutiérrez T., Raich, R. M., Sánchez, D. \& Deus, J. (2003). Instrumentos de evaluación psicológica de la salud. Madrid, España: Alianza. 
Harrison, K. \& Cantor, J. (1997). The relationship between media consumption and eating disorders. Journal of Communication, 47(1), 40-67.

Jáuregui, I., Romero, J., Bolaños, P., Montes, C., Díaz, R., Montaña, M. T., et al. (2009). Conducta alimentaria e imagen corporal en una muestra de adolescentes de Sevilla. Nutrición Hospitalaria, 24(5), 568-573.

Kanekoa, M. L. (2007). A correlation between the Eating Attitudes Test and Body Shape Questionnaire. Tesis de especialidad, Brigham Young University, Provo, Utah, Estados Unidos.

Kerlinger, F. N. \& Lee, H. B. (2002). Investigación del comportamiento (4a. ed.) (L. E. Pineda \& I. Mora, Trads.). México DF, México: McGraw-Hill. (Trabajo original publicado en 2000).

Keskín, G., Engin, E. \& Dulgerler, S. (2010). Eating attitude in the obese patients: the evaluation in terms of relational factors. Journal of Psychiatric and Mental Health Nursing, 17, 900-908.

Macedo, A., Soares, M. J., Azevedo, M. H., Gomes, A., Pereira, A. T., Maia, B., et al. (2007). Perfectionism and eating attitudes in Portuguese university student. European Eating Disorders Review, 15, 296-304.

Merino, H., Pombo, M. G. \& Godás, A. (2001). Evaluación de las actitudes alimentarias y la satisfacción corporal en una muestra de adolescentes. Psicothema, 13(4), 539-545.

Mintz, L. B. \& O'Halloran, M. S. (2000). The Eating Attitudes Test: Validation with DSM-IV eating disorder criteria. Journal of Personality Assessment, 74(3), 489-503.

Peláez, M. A., Labrador, F. J. \& Raich, R. M. (2007). Prevalence of eating disorders among adolescent and young adult scholastic population in the region of Madrid (Spain). Journal of Psychosomatic Research, 62, 681-690.

Pereira, A. T., Maia, B., Bos, S., Soares, M. J., Marques, M., Macedo, A., et al. (2008). The portuguese short form of the Eating Attitudes Test-40. European Eating Disorders Review, 16, 319-325.

Preti, A., Pinna, C., Nocco, S., Pilia, S., Mulliri, E., Micheli, V., et al. (2007). Rural/urban differences in the distribution of eating disorder symptoms among adolescents from community samples. Australian and New Zealand Journal of Psychiatry, 41, 525-535.

Rosabal, A. (2005). Riesgo de desórdenes alimentarios en adolescentes del Cantón Central de San José en noviembre del 2004 - abril del 2005. Tesis licenciatura, Universidad de Costa Rica, San José, Costa Rica.

Salazar, Z. (2008). Modelos televisivos y su relación con los trastornos de la conducta alimentaria, en adolescentes de ambos sexos del área metropolitana de San José. Tesis de maestría, Universidad de Costa Rica, San José, Costa Rica.

Smead, V. S. \& Richert, A. J. (1990). Eating Attitude Test factors in an unselected undergraduate population. International Journal of Eating Disorders, 9(2), 211-215.

Toro, J. \& Vilardell, E. (1987). Anorexia nerviosa. Barcelona, España: Martínez Roca.

Wegener, D. T. \& Fabrigar, L. R. (2000). Analysis and design for nonexperimental data: Addressing causal and noncausal hypotheses. En H. T. Reis \& C. M. Judd (Eds.), Handbook of research methods in social and personality psychology (pp. 412-450). New York: Cambridge University Press. 
Vázquez, A. J., García-Bóveda, R. J. \& Vázquez-Morejón, R. (2007). Características psicométricas de una adaptación española del Test de Bulimia (BULIT). Actas Esp Psiquiatr, 35(5), 309-314.

Zeledón, M. J. (2004). The weight of support: Communication of social support for women diagnosed with bulimia. Tesis de maestría, Universidad del Estado de San Diego, San Diego, Estados Unidos.

Recibido: 08 de febrero de 2011

Aceptado: 24 de enero de 2012 